\title{
The Determination of Stress State of Structures Considering Sequence of Construction and Load Application
}

\author{
Vladimir Kuroedov ${ }^{1}$, Luka Akimov ${ }^{1,},{ }^{*}$, Artem Frolov ${ }^{1}$, Alexey Savchenko ${ }^{1}$, Anton \\ Kuznetsov $^{1}$, and Aleksandr Kostenko ${ }^{1}$ \\ ${ }^{1}$ Peter the Great Saint-Petersburg Polytechnic University, 29 Polytechnicheskaya st., St.Petersburg, \\ 195251, Russian Federation
}

\begin{abstract}
For more accurate calculation of structure it is required to apply loads in the process of its construction instead of load application after structure has taken its final form. This action is necessary in order to observe changes in the stress-strain state of the structure under study. This circumstance is important for the massive hydraulic structures, such as hydroelectric dams. It is required to determine tensions, deformations and displacements in solving the building-up tasks. It is necessary to consider the problem for constructions of linear and nonlinear materials and check the principle of superposition on which the method of solving nonlinear problems is based. Also it is necessary to consider the solution of finite element schemes with the help of various iterative methods, such as the method of additional loads and the method of variable.
\end{abstract}

\section{Introduction}

It is usually assumed in the static calculation of structures that load application is carried out after the structure has taken its final form [1]. Changes of the stress-strain state of structures are mostly investigated only with the appearance of various defects in the construction that could occur during the construction process [2-6].

However, a number of authors show that it is necessary to take into account the following circumstance: the load is applied during the erection of structure, the stress-strain state changes until the construction gets its final form and loads get their final quantity [7$11]$.

It is particularly important to pay attention to this circumstance during the calculation of massive structures [12]. These requirements have been included in EC [13, 14].

\footnotetext{
*Corresponding author: lukas-ak@mail.ru
} 


\section{Materials and Methods}

The solution of the building-up tasks $[8,9]$ is leaded to the determination of tension $\bar{\sigma}$, deformation $\bar{\varepsilon}$ and displacement $\overline{\mathrm{u}}$ in the finally formed body depending on its configuration changes, characterized by the k-phase of the building-up task by the quantities $\bar{\Omega} \bar{S}^{(k)}, \bar{S}^{(k)}$ and the sequence of the load application $P^{(k)}$. Thus, in case of linear task (when $\sigma=D \varepsilon$ ), for the weighty body:

$$
\bar{\Omega}^{(N)}=\sum_{k=1}^{N} \omega^{(k)}
$$

which is constructed by layers of finite thickness $\omega^{(k)}$, expressions for the quantities $\overline{\sigma_{i}}$ $\overline{\varepsilon_{i}}, \overline{u_{i}}$, appearing in i-th layer, would have the following form:

$$
\begin{aligned}
& \bar{\sigma}_{i}=\sum_{k=1}^{N} \sigma_{i}^{(k)} \\
& \bar{\varepsilon}_{i}=\sum_{k=1}^{N} \varepsilon_{i}^{(k)} \\
& \bar{u}_{i}=\sum_{k=1}^{N} u_{i}^{(k)}
\end{aligned}
$$

Here: $\mathrm{N}$ - the number of building-up layers; $\mathrm{k}$ - the number of stage of the building-up when the structure gets the $\bar{\Omega}^{(k)}$ form and the forces of tension, deformation and displacement come into effect $P^{(k)}: \sigma_{i}^{(k)}, \varepsilon_{i}^{(k)}, u_{i}^{(k)}$. These forces appear in the i-th layer in the process of solution of the tasks with fixed borders $\bar{S}^{(k)}$ of the linear theory of elasticity for the computational region $\bar{\Omega}^{(k)}$ during the action of dead load of the layer $\omega^{(k)}-G^{(k)}$, as $G^{(k-1)}=G^{(k-2)}=\ldots=G^{(k)}=0$. At the same time, the other components of the load $P^{(k)}$ are calculated in the same way and their quantities and points of application must be known for every stage of the building-up.

We shall consider the solution of the same problem for the structure made of nonlinear material. The law of elasticity of such material in the general case is written as:

$$
\sigma=D(\varepsilon) \varepsilon
$$


where $D(\varepsilon)$ is the matrix of elasticity which components are situated on the basis of the adjusted link between the invariants of the tensor of tensions $\sigma_{0}, \sigma_{i}$ and the tensor of deformations $e_{0}, e_{i}$ :

$$
\begin{aligned}
& \sigma_{0}=f_{1}\left(e_{0}, e_{i}\right) \\
& \sigma_{i}=f_{2}\left(e_{0}, e_{i}\right)
\end{aligned}
$$

The solution of the tasks that were mentioned above is complicated by the following circumstance: the principle of superposition could not be used in this case.

\section{Result and Discussion}

It is necessary to derive the equilibrium equation in terms of displacements. The solution of the $\mathrm{u}_{\mathrm{k}}$ task with fixed boundaries on the $\mathrm{k}$-th stage of the calculation for the area $\Omega^{(k)}=\Omega^{(k-1)}+\omega^{(k)}$ with fixed boundaries $S^{(k)}$ should satisfy this equilibrium equation. In this case we should assume the following statement: the final quantities of tensions that were received on the (k-1)-th and k-th stages of the calculation must satisfy the law of elasticity Eq.5 and the balance equation:

$$
A^{\prime} \bar{\sigma}+P=0
$$

The expressions for the tension $\bar{\sigma}_{\mathrm{k}}$ could be represented as follows:

$$
\bar{\sigma}_{k}=D\left(\bar{\varepsilon}^{*}\right) \bar{\varepsilon}^{*}+\bar{D}\left(\varepsilon, \bar{\varepsilon}^{*}\right) \varepsilon_{k}
$$

where $\bar{\varepsilon}^{*}$ - the deformation which has occurred in the building-up body before the installation of the $\omega^{(k)}$ layer, $\varepsilon_{k}=A u_{k} ; \bar{D}\left(\varepsilon, \bar{\varepsilon}^{*}\right)$ - the reduced matrix of elasticity which components depend not only on the solution of the $\mathrm{u}_{\mathrm{k}} \mathrm{task}$, but also on the deformations $\bar{\varepsilon}^{*}$ which have already taken place in the building-up body before the installation of the layer.

Substituting Eq.9 into Eq.8 that corresponds to the k-th stage of the erection of array and considering that a similar equation holds for the (k-1)-th stage of the building-up task, we could receive the equilibrium equation in terms of displacements which must satisfy the solution of the task with fixed boundaries $S^{(k)}$ :

$$
A^{\prime} \bar{D}\left(\varepsilon, \bar{\varepsilon}^{*}\right) A u_{k}+P_{k}=0
$$

In this expression $\mathrm{P}_{\mathrm{k}}$ corresponds to the load of the re-stacking layer $\omega^{(k)} \cdot \sigma^{(k)} \cdot\left(\sigma^{(k-1)}=\ldots=\sigma^{(1)}=0\right)$ and to the forces applied to the construction at this stage of building-up.

Substituting Eq.5 into Eq.9 and considering that $\bar{\varepsilon}_{k}=\bar{\varepsilon}^{*}+\varepsilon_{k}$, we could obtain the expression for the calculation of components of the reduced matrix of elasticity: 


$$
\bar{D}\left(\varepsilon, \bar{\varepsilon}^{*}\right)=\left[D\left(\bar{\varepsilon}^{*}+\varepsilon\right)-\left[D\left(\bar{\varepsilon}^{*}\right)-D(\bar{\varepsilon} *+\varepsilon)\right] \bar{\varepsilon} * \cdot \frac{\varepsilon^{\prime}}{|\varepsilon|^{2}}\right]
$$

where $|\varepsilon|=\sqrt{\varepsilon^{\prime} \varepsilon}$.

Thus, the solution of the building-up task for the body $\bar{\Omega}$ constructed from the material, which behavior is described by the nonlinear law Eq.5, reduces to the solution of the tasks with fixed boundaries $S^{(k)}(\mathrm{k}=1,2, \ldots, \mathrm{N})$ concerning to the Uk sequence. The behavior of the material in each task is described by the nonlinear law:

$$
\sigma=\bar{D}\left(\varepsilon, \bar{\varepsilon}^{*}\right) \varepsilon
$$

with the asymmetrical matrix $\bar{D}\left(\varepsilon, \bar{\varepsilon}^{*}\right)$, defined according to Eq.11.

In the case of simple connection between tension and deformation (for instance, for incompressible media) the law of elasticity Eq.11 could be interpreted as the original Eq.5 and written in the new system of coordinates $\bar{\sigma}-\bar{\varepsilon}$ with the beginning that corresponds to quantities $\bar{\sigma}^{*}$ and $\bar{\varepsilon}^{*}$. Similar considerations could be used for more general cases of the construction of buildings.

In solving tasks by finite-element method [15] we also solve the nonlinear task with the fixed boundaries Sk on the each stage of the building-up:

$$
\bar{K}(q)^{(k)} q^{(k)}=P^{(k)}, \mathrm{k}=1,2, \ldots, \mathrm{N}
$$

The expression for the calculation of the final values of the stresses in the elements $r \in \omega^{(i)}$ would take the following form:

$$
\left\{\bar{\sigma}^{r}\right\}_{i}=D(\varepsilon) \cdot\left\{\bar{\varepsilon}^{r}\right\}_{i}
$$

where

$$
\left\{\bar{\varepsilon}^{r}\right\}_{i}=B^{r}\left\{\bar{q}^{r}\right\}_{i}
$$

and

$$
\left\{\bar{q}^{r}\right\}_{i}=\sum_{k=i}^{N}\left\{q^{r}\right\}_{i}^{(k)}
$$

It is necessary to mark that using similar considerations in case of the linear formulation we would come to the known scheme of solution of the building-up tasks.

Problems of type Eq.13 are usually solved using various iterative methods. In this case the method of the additional loads is more suitable, because the methods of the variable parameters are unsuitable due to the peculiarities of the matrix $\bar{D}\left(\varepsilon, \bar{\varepsilon}^{*}\right)$. 
Let us consider one option of the iterational process when the system of linear algebraic equations Eq.13 with the symmetric stiffness matrix of the element system is solved $r \in \Omega^{(k)}[16]:$

$$
K^{(k)} q_{j+1}^{(k)}=P^{(k)}-\bar{L}_{j}^{(k)}, \mathrm{j}=1,2, \ldots, \mathrm{n}
$$

Here $\bar{L}_{j}^{(k)}$ is the vector of the generalized nodal forces, caused by the nonlinear coupling Eq.12 between the tension and deformation:

$$
\begin{gathered}
\left(\bar{L}_{m}\right)_{j}^{(k)}=\sum_{r \in m}\left\{\left(\bar{L}_{m}\right)_{j}^{r}\right\}^{(k)} \\
\left\{\left(\bar{L}_{m}\right)_{j}^{r}\right\}^{k}=\int_{V^{\prime}}\left\{B^{r}\right\}^{\prime} \cdot\left\{\left\{\bar{D}\left(\varepsilon, \bar{\varepsilon}^{*}\right)_{j}^{r}-D^{r}\right\} \varepsilon_{j}^{r}\right\}^{(k)} d v
\end{gathered}
$$

where $D^{r}$ is the stiffness matrix of the element $\mathrm{r}$, that corresponds to the initial approximation $(\mathrm{j}-0)$.

\section{Conclusions}

By using this method it becomes possible to solve the task considering the geometrical nonlinearity. This method allows to solve stability problems of elements and constructions [17-20].

The method of solving tasks with the loads application to a building in the process of its construction or the method of up-building has demonstrated sufficient efficacy in the calculation of structures [21]. In solving these tasks it is necessary to take into account both the nonlinear behaviour of the material and the sequence of construction of buildings [22, 23]. It is especially important to consider these two factors in the calculation of massive hydraulic structures in which the stress-strain state changes as long as the structure has not obtained its final form. It would be especially relevant by using this method in a variety of computer-aided calculation complexes of building design in solving planar linear and physical nonlinear problems of up-building. In these tasks the load will be applied during the construction of buildings whereby it will be possible to observe the change of the stressstrain state of the facilities. It is also appropriate to explore this circumstance in a calculation of other industrial and civil buildings in which it is necessary to take into account the sequence of construction and the application of loads.

\section{References}

1. Yousuf Dinar, Munshi Md. Rasel, Muhammad Junaid Absar Chowdhury, Md. Abu Ashraf, The Int. J. Of Eng. And Sc, 3(1), 52-63 (2013)

2. O. V. Kabantsev, S. V. Gorbatov, K. O. Pesin, Vestnik TSUAB. English ver. app. to No. 6, 1-2, 11-25 (2015)

3. O.V. Kabantsev, Proc. 3rd All-Rus. Conf. on Conc. and Reinf. Conc., 1, 282-292 (2014)

4. O.V. Kabantsev, A.V. Karlin, J. Ind. and Civil Eng., 7, 33-35 (2012)

5. O.V. Kabantsev, A. Perelmuter, Proc. Eng., 57, 479-488 (2013) 
6. N.D. Sergeev, Stroit. mekh. i raschet sooruzhenii., 6, 11-16 (1975)

7. S. Albanesi, N. Antolini, S. Biondi, The influence of building phases in the post-elastic behavior of multistoried reinforced concrete frames (11th World Conference on Earthquake Engineering, Acapulco, 1996)

8. S.G. Shulman, Raschety gydrotehnicheskih sooruzhenyi s uchetom posledovatelnosty vozvedeniya (Energia, Moscow, 1975)

9. L.I. Dyatlovickiy, A.I. Raynberg, Formirovaniye napryazheniy v gravitacuonnih plotinah (Naukova Dumka, Kiev, 1975)

10. H.L. Yip, F.T.K. Au, S.T. Smith, Proc. Eng., 14, 1384-1391 (2011)

11. V. V. Kuroedov, Trudy LPI. 363, 70-73 (1997)

12. Bondarev, A., Yugovb, A., Proc. Eng., 117, 948-958 (2015)

13. D. Zejak, N. Vatin, V. Murgul, Appl. Mech. and Mat., 725-726, 111-117 (2015)

14. Z. Zafirovski, N. Vatin, Appl. Mech. and Mat., 725-726, 214-219 (2015)

15. L.A. Rozin, Raschet gydrotehnicheskih sooruzhenyi na ECVM. Metod konechnih elementov (L: Energia, Leningrad, 1971)

16. V.V. Kuroedov, Trudi LPI., 349, 43-47 (1976)

17. V.V. Lalin, D.A. Kushova, Int. j. for comp. civ. and str., 4(9), 178-185 (2013)

18. V.V. Lalin, A.V. Yavarov, 5, 51-54 (2013)

19. V. Lalin, E. Zdanchuk, Int. J. Of Math. Mod. and Meth. In Appl. Sc., 8, 208-123 (2014)

20. V. Lalin, D. Kushova, Variational formulations of the nonlinear equilibrium and stability problems of elastic rods (International Conference „Innovative Materials, Structures and Technologies", Tallinn, 2014)

21. M. Dabaon, E. Ellobody, K. Ramzy, J. of Constr. Steel Res., 110, 16-28 (2015)

22. Sayed Behzad Talaeitabaa, Minoo Halabiana, Mohammad Ebrahim Torkib, ThinWalled Struct., 95, 389-407 (2015)

23. O.A. Rosenboom, T.F. Paret, G.R. Searer, Chronological Construction Sequence, Creep, Shrinkage, and Pushover Analysis of an Iconic 1960s Reinforced Concrete Building (15th World Conference on Earthquake Engineering, Lisboa, 2012) 